\title{
Electrostatic self-assembly of multilayer copolymeric membranes on the surface of porous tantalum implants for sustained release of doxorubicin
}

\author{
This article was published in the following Dove Press journal: \\ International Journal of Nanomedicine \\ 25 November 2011 \\ Number of times this article has been viewed
}

\author{
Xinming Guo ${ }^{1, *}$ \\ Muwan Chen ${ }^{1,2, *}$ \\ Wenzhou Fengl,* \\ Jiabi Liang' \\ Huibin Zhao' \\ Lin Tian' \\ Hui Chao ${ }^{3}$ \\ Xuenong Zou' \\ 'Orthopaedic Research institute/ \\ Department of Orthopaedic Surgery, \\ the First Affiliated Hospital and \\ Department of Pharmacy, the Fifth \\ Affiliated Hospital, Sun Yat-sen \\ University, Guangzhou, People's \\ Republic of China; ${ }^{2}$ Interdisciplinary \\ Nanoscience Center (iNANO), \\ Aarhus University, Denmark; \\ ${ }^{3}$ Ministry of Education Key \\ Laboratory of Bioinorganic and \\ Synthetic Chemistry, School of \\ Chemistry and Chemical Engineering \\ of Sun Yat-sen University, Guangzhou, \\ People's Republic of China
}

*The first three authors contributed equally to this work as co-first author

Correspondence: Xuenong Zou

Orthopaedic Research Institute/

Department of Orthopaedic Surgery, the

First Affiliated Hospital of Sun Yat-sen

University, 510080 Guangzhou, People's

Republic of China

Tel +862087755766

Fax +86 2087332150

Email zxnong@hotmail.com

\begin{abstract}
Many studies in recent years have focused on surface engineering of implant materials in order to improve their biocompatibility and other performance. Porous tantalum implants have increasingly been used in implant surgeries, due to their biocompatibility, physical stability, and good mechanical strength. In this study we functionalized the porous tantalum implant for sustained drug delivery capability via electrostatic self-assembly of polyelectrolytes of hyaluronic acid, methylated collagen, and terpolymer on the surface of a porous tantalum implant. The anticancer drug doxorubicin was encapsulated into the multilayer copolymer membranes on the porous tantalum implants. Results showed the sustained released of doxorubicin from the functionalized porous tantalum implants for up to 1 month. The drug release solutions in 1 month all had inhibitory effects on the proliferation of chondrosarcoma cell line SW1353. These results suggest that this functionalized implant could be used in reconstructive surgery for the treatment of bone tumor as a local, sustained drug delivery system.
\end{abstract}

Keywords: self-assembly, surface modification, tantalum, drug delivery system, doxorubicin, bone tumor

\section{Introduction}

Bone tumors (either primary or secondary) are one of the most severe diseases in orthopedic clinical practice. The common approach for bone tumors is a combination of surgery, chemotherapy, and radiology. Reconstructive surgery for bone cancer patients requires precise removal of the affected tissue and replacement with a bone implant followed by postoperational chemotherapy. ${ }^{1,2}$

Metallic implants using titanium and tantalum have been widely used due to their superior mechanical strength, physical stability, and biocompatibility. ${ }^{3-7}$ Basic scientific and clinical studies have shown that osseointegration is a well-established property of porous tantalum implant, ${ }^{8-10}$ which is promising for reconstructive surgeries. ${ }^{4,11}$

On the other hand, systemic chemotherapy is the most commonly applied strategy of postoperational chemotherapy. The effectiveness of systemic chemotherapy has been improved; however, there are still limitations in clinical practice, including a number of side effects, such as renal damage, liver damage, cardiomyopathy, and myelosuppression, which may necessitate the suspension of treatment. Furthermore, systemic chemotherapy is not efficient, because the concentration of drug is diluted at the targeted site. Therefore, local drug delivery systems have received increasing interest in recent years. In 1997, Fröschle et al ${ }^{12}$ reported that 
daunorubicin-polymethylmethacrylate in the resection cavity delayed or reduced recurrences in bone metastasis in animals. Recently, El-Ghannam et al tested a ceramicbased anticancer drug, 5-fluorouracil, to treat breast cancer in a murine model. ${ }^{13}$ To the best of our knowledge, there is still not an implantable drug delivery system available on the market for bone tumor patients. Researchers are still trying to design a multifunctionalized implant to not only support the skeletal structure but also provide local, controllable release of an anticancer drug in order to prevent cancer recurrence. ${ }^{14,15}$

In the current study we intended to functionalize the surface of the porous tantalum implant so as to have a sustained drug delivery capability via electrostatic self-assembly of polyelectrolytes of hyaluronic acid, methylated collagen, and terpolymer of hydroxylethyl methacrylate-methyl methacrylate-methylacrylic acid (HEMA-MMA-MAA). The anticancer drug doxorubicin (DOX) was encapsulated into the multilayer copolymer membranes on the porous tantalum implants. Previously, we developed this electrostatic self-assembly technique for the improvement of osteogenic differentiation of mesenchymal stem cells and calcium deposition, which are important parameters of bone tissue engineering. ${ }^{16}$ Here, we focused on the drug release aspect and the bioactivity test of the loaded drug DOX. Firstly, we optimized the self-assembly membranes to obtain the optimal drug release rate by the use of an orthogonal design table. Then, we monitored the drug release rate from the implants for 1 month. Finally, we tested the bioactivity of DOX, which was released from functionized porous tantalum implants, by the inhibition of chondrosarcoma cell line SW1353. We aimed at adding a drug delivery capability to the reconstructive porous tantalum implant.

\section{Materials and methods}

The porous tantalum implants, Trabecular Metal ${ }^{\mathrm{TM}}$ (Ta), were supplied from Zimmer Inc. (Minneapolis, MN). They are made by chemical vapor deposition of tantalum precursors on a vitreous carbon skeleton. The average pore size of the tantalum implant is 430 microns, and the porosity is $75 \%-$ $80 \%$. Hyaluronic acid (780 kDa, Lot P9805-9A) was purchased from Lifecore Biomedical Inc. (Chaska, MN) HEMA, MMA, and MAA were purchased from Sigma-Aldrich, Shanghai, China. DOX standard was purchased from Wanle, Shenzhen, China. The collagen (mainly bovine collagen type I) was courtesy of Professor Yinjun Wang, Materials and Engineer College, South China University of Technology, China.

\section{Materials preparation \\ Collagen methylation}

Type I bovine collagen was modified to be cationic via esterification, as described previously. ${ }^{17}$ The degree of methylation was controlled by adjusting the time and temperature of the reaction. ${ }^{18}$ The precipitated collagen type I was dissolved in $0.1 \mathrm{M} \mathrm{HCl}$ containing methanol for 6 days at $4^{\circ} \mathrm{C}$. At the end of the reaction, the solution was dialyzed against deionized water at $4^{\circ} \mathrm{C}$ using dialysis tubing with molecular weight cut-off (MWCO) of 8000-15,000 until the $\mathrm{pH}$ of the external reservoir reached 6.4, followed by freeze-drying. The modified collagen was stored at $-20^{\circ} \mathrm{C}$ for use.

\section{Terpolymer preparation}

Terpolymer of HEMA, MMA, and MAA was synthesized by solution polymerization at $78^{\circ} \mathrm{C}$ in 2-propanol using $2,2^{\prime}$ azobisisobutyronitrile (AIBN) as an initiator, as described previously. ${ }^{17}$ The themolar feed ratio of HEMA, MMA, and MAA was 25:50:25. Terpolymer 3\% and 10\% in phosphatebuffered saline solution was used in this study.

\section{Surface modification}

\section{Pretreatment of porous tantalum implants}

The porous tantalum implants were placed in the $0.1 \mathrm{M}$ of $\mathrm{NaOH}$ solution for 24 hours at $60^{\circ} \mathrm{C}$. Then, the implants were rinsed with distilled water five times and dried in an electric oven at $80^{\circ} \mathrm{C}$. After this pretreatment, the implants were used for surface modification and drug loading.

\section{Assembly of membranes}

Porous tantalum implants were immersed in the hyaluronic acid solution for 30 minutes and then freeze-dried. A mixed solution of $100 \mu \mathrm{L}$ of methylated collagen with DOX was added into the hyaluronic acid precoated porous tantalum implant and dried in the desiccator in a vacuum for 30 minutes. The final coating layer was done by pipetting $100 \mu \mathrm{L}$ of copolymer solution into the precoated porous tantalum implants, which were left at room temperature for 1 hour and then dried in the desiccator under vacuum. The implant functional procedure is shown in Figure 1.

Scanning electron microscopy (SEM, Nova NanoSEM 600 , FEI Company, Eindhoven, The Netherland) was used to visualize the morphology and distribution of the coating on the tantalum implant.

\section{The orthogonal experimental design for optimization of sustained drug release} We optimized the self-assembly membranes to obtain the optimal drug release rate by the use of an orthogonal 


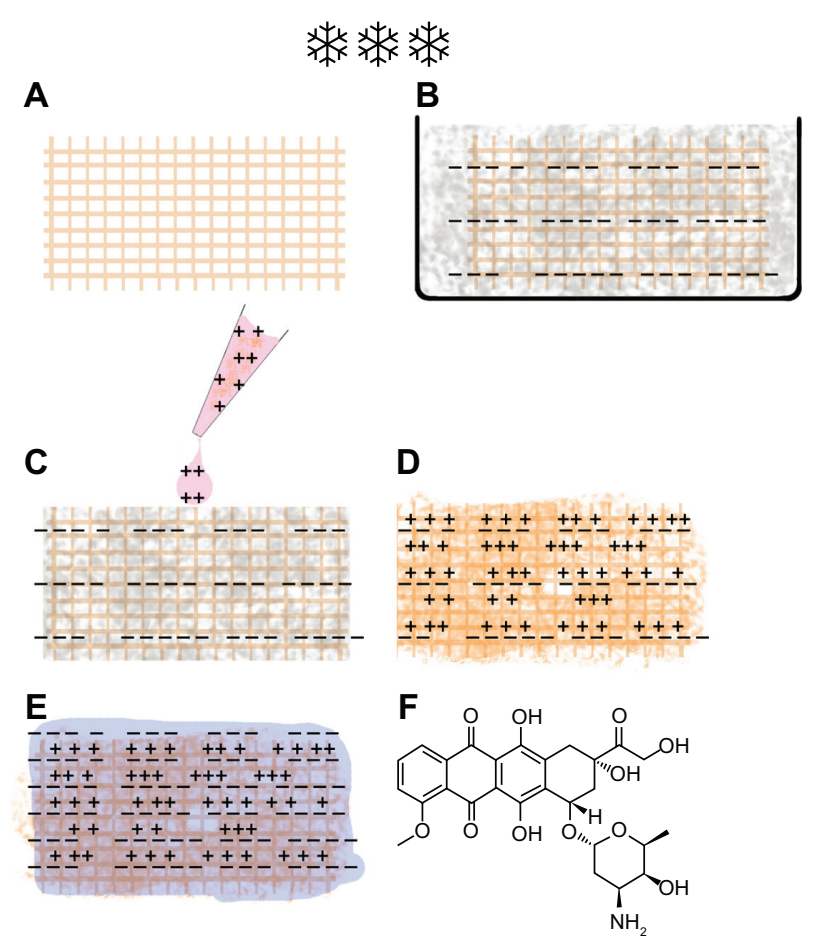

Figure I Procedure of implant functionalization and doxorubicin (DOX) loading. (A) A macroporous tantalum implant. (B) Implant embedded with hyaluronic acid followed with lyophilization. (C) DOX mixed with methylated collagen was dropped on to the embedded implant. (D) A hyaluronic acid-embedded implant was loaded with DOX and methylated collagen. (E) Terpolymer of hydroxylethyl methacrylatemethyl methacrylate-methylacrylic acid was added into the previous functiona implant (D) and an implant with the copolymeric multilayer membranes loaded with DOX was made. "_-_ represents negative charge, and "+++" represents positive charge. (F) The chemical structure of DOX.

design table. Three parameters, including the concentrations of hyaluronic acid (A), methylated collagen (B), and terpolymer $(\mathrm{C})$, were defined as main factors. Nine formulations were designed for the test according to the standard $\mathrm{L}_{9}\left(3^{3}\right)$ orthogonal experimental design, in order to screen the optimal formulations of prescription. Cumulative DOX release rates $(\mathrm{Q})$ from porous tantalum implants at 2 hours and 30 days were selected as indexes to determine the optimal factors. Experiments have been orthogonally designed for arranging the three factors with three levels (for A and B) and two levels (for C), as shown in Table 1.

\section{In vitro drug release test}

The determination of DOX by chromatography

The high-performance liquid chromatography system consisted of the 1200 Series lsocratic pump (Agilent Corporation, CA), a manual injector (20 mL loop), an Agilent 1200 Series-fluorescent detector, and Agilent Technologies chemstation. The separation was carried out on a $4.6 \times 150 \mathrm{~mm}(5 \mu \mathrm{m})$ reversed-phase C18 Luna column. The mobile phase consisted of ( $5 \%$ phosphoric acid) water:methyl cyanides:methanol:isopropyl alcohol
Table I Levels of each of the three factors for the sustained drug release $(\mathrm{mg} / \mathrm{mL})$

\begin{tabular}{llll}
\hline Factors & Level I & Level 2 & Level 3 \\
\hline Hyaluronic acid (A) & $\mathrm{I}$ & 2 & 4 \\
Methylated collagen (B) & $\mathrm{I} .5$ & 3 & 4.5 \\
Terpolymer (C) & 30 & 100 & \\
\hline
\end{tabular}

(65:15:10:10, v:v:v). The flow rate was maintained at $1 \mathrm{~mL} /$ minute. The effluents were monitored at $\lambda \mathrm{Ex}=505 \mathrm{~nm}$ and $\lambda \mathrm{Em}=550 \mathrm{~nm}$. All chromatographic analyses were performed at room temperature.

\section{Standard solutions}

DOX $(0.05-10 \mu \mathrm{g} / \mathrm{mL})$ was prepared in the mobile phase. DOX $(0.0010 \mathrm{~g})$ was dissolved in a $5 \mathrm{~mL}$ mobile phase in a volumetric flask. Various standard solutions were then prepared from this stock solution after adequate dilution with a mobile phase.

\section{In vitro DOX release from the implants}

The release profile of DOX from porous tantalum implants was determined by incubating an implant in $2.0 \mathrm{~mL}$ stimulated body fluid in a shaking water bath at $37^{\circ} \mathrm{C}$ for 1 month. At the series time points of 0.33 hours, 0.67 hours, 1 hour, 2 hours, 6 hours, 12 hours, 24 hours, 48 hours, 96 hours, 144 hours, 15 days, and 30 days, $2 \mathrm{~mL}$ of solution was collected and replaced with $2 \mathrm{~mL}$ of fresh stimulated body fluid. The drug concentrations of samples were measured by high-performance liquid chromatography, and the cumulative release rates were calculated afterwards.

\section{In vitro cytotoxicity test of DOX released from porous tantalum implant}

Chondrosarcoma cell line SW1353 was seeded in 96-well plates at a density of 2000 cells/well in each well with $200 \mu \mathrm{L}$ Dulbecco's Modified Eagle Medium containing $10 \%$ fetal calf serum. After 2 days' culture, $10 \mu \mathrm{L}$ of sterile-filtered DOX-released solutions from series time points of 2 hours, 24 hours, 15 days, and 30 days were added into the culture medium. Cells that were cultured in stimulated body fluid were used as control. Each treatment had six replicates. After 3 days, SW1353 cells were collected for viability testing determined by MTT assay. Briefly, to each well was added $10 \mu \mathrm{L}$ of MTT stock solution $(5 \mathrm{~g} / \mathrm{L})$, which was cultured at $37^{\circ} \mathrm{C}$ for 4 hours. Media were removed and the converted dye was dissolved in $100 \mu \mathrm{L}$ of DMSO solution. Absorbance of converted dye was 
measured at a wavelength of $490 \mathrm{~nm}$. Cell inhibitory rate was calculated as $\% \mathrm{IC}=\left(1-\mathrm{OD}_{\text {testing }}\right.$ group $\left./ \mathrm{OD}_{\text {control }}\right) \times 100 \%$.

\section{Results and discussion Characterization of surface modification by SEM}

From the SEM micrographs (Figure 2) we could observe that the porous tantalum implant has a trabecular bone-like structure with highly interconnected pores, ranging from $200 \mu \mathrm{m}$ to $400 \mu \mathrm{m}$ (Figure 2A). We chose a representative position of the porous tantalum implant (Figure 2B) to characterize the modification procedure. After the initial layer coating of hyaluronic acid, nanofibres of hyaluronic acid were observed on the surface of the trabacula as well as the pores (Figure 2C). After adding methylated collagen and terpolymer, we found that a hyaluronic acid-methylated collagen-terpolymer complex was formed within the porous tantalum implant. The copolymer membranes were uniform, covering the surface of porous tantalum trabecula, and did not block the porous structures (Figure 2D).

The copolymer membranes formed on the surfaces of the porous tantalum implant occurred by electrostatic self-assembly of oppositely charged polyelectrolytes. When negative and positive polymers are mixed, charge-charge interactions result in the formation of a polyelectrolyte complex. This technique has been widely applied in drug delivery systems ${ }^{19}$ and tissue engineering devices. ${ }^{17,20}$ Quek et $\mathrm{al}^{21}$ used methylated collagen and terpolymer of
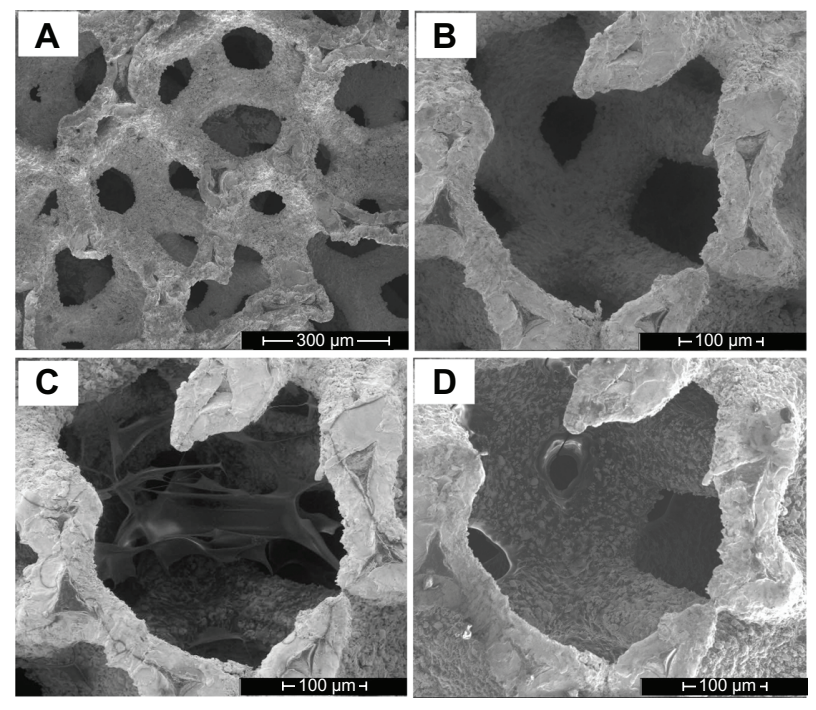

Figure 2 Scanning electron microscopy micrographs of the porous tantalum implant. (A) The bare porous tantalum implant with highly interconnected pores. (B) A representative position before surface modification. (C) The representative position after hyaluronic acid modification. (D) The representative position after hyaluronic acid, methylated collagen, and terpolymer modification.
HEMA-MMA-MAA to form a two-layered membrane for encapsulation of hepatocytes. In our previous study we used hyaluronic acid, methylated collagen, and terpolymer of HEMA-MMA-MAA to form a composite matrix for functionalization of bone tissue engineering scaffolds. ${ }^{16}$

In this study, porous tantalum implants were pretreated with $\mathrm{NaOH}$ to obtain an amorphous sodium tantalate layer on the surface to increase the bioactivity of the implant. ${ }^{22}$ Due to the viscosity of hyaluronic acid solution, there were networks of hyaluronic acid nanofibres covering the trabecular implant after the freeze-dryer procedure. The porous tantalum implant was embedded in the hyaluronic acid and the surface area of the implant was increased, so the implant was finally embedded in the membranes after the electrostatic assembly among hyaluronic acid, methylated collagen, and terpolymer of HEMA-MMA-MAA. This was different from the study carried out by Toh et al, ${ }^{23}$ which used methylated collagen and terpolymer to macroencapsulate a whole implant.

For the drug loading study, DOX was mixed in the methylated collagen solution, which was because DOX dispersed better in the collagen solution compared with in the hyaluronic acid solution, and also avoided direct contact of DOX with the porous tantalum metal surface. Because the chemical structure of DOX (Figure 1F) contains an amino group with a $\mathrm{pKa}$ of 8.6, it is positively charged at $\mathrm{pH} 7.4$. DOX can interact with the polyanions, either with hyaluronic acid or with the terpolymer of HEMA-MMA-MAA, and form polymer-drug complexes due to electrostatic interaction between oppositely charged polyions. In this study, DOX served as both a coating component and a functional drug.

Polyelectrolytes and polyelectrolyte complexes have been extensively studied in the development of drug delivery as drug carriers or substances. The polyelectrolyte complexes can be polymer-polymer, polymer-drug, and polymer-drug-polymer. Drug-carrying polyelectrolyte microparticles have been shown to prolong drug release time. ${ }^{24}$ Polyelectrolyte multilayer nanofilms have been studied for delivery of charged dyes, metal and inorganic nanoparticles, DNA, proteins, and viruses. ${ }^{19}$

\section{Determination of the optimal formulations of prescription}

The formulation factors affecting cumulative DOX release rates were studied in an orthogonal experimental design. The results are listed in Table 2 (DOX release rate at 2 hours) and Table 3 (DOX release rate at 30 days). 
Table 2 Results of the test of orthogonal design of cumulative doxorubicin release rates (QI) from the functionalized porous tantalum implants at 2 hours

\begin{tabular}{lllll}
\hline & A $^{\mathrm{a}}(\mathbf{m g} / \mathbf{m L})$ & $\mathbf{B}^{\mathrm{b}}(\mathbf{m g} / \mathbf{m L})$ & $\mathbf{C}^{\mathrm{c}}(\mathrm{mg} / \mathbf{m L})$ & Q I (\%) \\
\hline $\mathrm{I}$ & $\mathrm{I}$ & $\mathrm{I}$ & $\mathrm{I}$ & $8.6 \mathrm{I}$ \\
2 & $\mathrm{I}$ & 2 & $\mathrm{I}$ & 23.6 \\
3 & $\mathrm{I}$ & 3 & 2 & 55.0 \\
4 & 2 & $\mathrm{I}$ & 2 & 22.0 \\
5 & 2 & 2 & $\mathrm{I}$ & 27.0 \\
6 & 2 & 3 & $\mathrm{I}$ & 52.2 \\
7 & 3 & $\mathrm{I}$ & $\mathrm{I}$ & 24.6 \\
8 & 3 & 2 & 2 & 28.6 \\
9 & 3 & 3 & $\mathrm{I}$ & 54.0 \\
$\mathrm{~K} \mathrm{I}^{\mathrm{d}}(\%)$ & 29.0 & $\mathrm{I} 8.4$ & $3 \mathrm{I} .7$ & \\
$\mathrm{~K} 2(\%)$ & 33.7 & 26.4 & 35.2 & \\
$\mathrm{~K} 3(\%)$ & 35.7 & 53.7 & & \\
$\mathrm{R}^{\mathrm{e}}(\%)$ & 6.7 & 35.3 & 3.5 & \\
\hline
\end{tabular}

Notes: ${ }^{\mathrm{a}} \mathrm{A}$ is the hyaluronic acid solution concentration; ${ }^{\mathrm{b}} \mathrm{B}$ is the methylated collagen concentration; ' $\mathrm{C}$ is the copolymer concentration; ${ }^{\circ} \mathrm{K}$ is the average value of the factor; ${ }^{e} \mathrm{R}$ is the range.

The effects of each factor were analyzed by comparing the $\mathrm{K}$ (average value of $\mathrm{Q}$ of each factor at same level) value and the range $\mathrm{R}\left(\mathrm{K}_{\max }-\mathrm{K}_{\min }\right)$. The range reflected the extent of each factor effect on index. With a bigger range, the extent affected was greater.

\section{Effects of each factor on the cumulative DOX release rate from the functionalized porous tantalum implants at 2 hours}

According to the $\mathrm{R}$ values, three factors in the present experiment were ranged as $\mathrm{B}>\mathrm{A}>\mathrm{C}$ (Table 2). To avoid a burst release at the early time point, a lower Q1 is better for

Table 3 Results of the test of orthogonal design of cumulative doxorubicin release rates (Q2) from the functionalized porous tantalum implants at 30 days

\begin{tabular}{lllll}
\hline & A $^{\mathrm{a}}(\mathbf{m g} / \mathbf{m L})$ & $\mathbf{B}^{\mathrm{b}}(\mathbf{m g} / \mathbf{m L})$ & $\mathbf{C}^{\mathrm{c}}(\mathbf{m g} / \mathbf{m L})$ & Q2 (\%) \\
\hline $\mathrm{I}$ & $\mathrm{I}$ & $\mathrm{I}$ & $\mathrm{I}$ & 18.6 \\
2 & $\mathrm{I}$ & 2 & $\mathrm{I}$ & 46.1 \\
3 & $\mathrm{I}$ & 3 & 2 & 81.0 \\
4 & 2 & $\mathrm{I}$ & 2 & 29.2 \\
5 & 2 & 2 & $\mathrm{I}$ & 36.6 \\
6 & 2 & 3 & $\mathrm{I}$ & 18.6 \\
7 & 3 & $\mathrm{I}$ & $\mathrm{I}$ & 28.9 \\
8 & 3 & 2 & 2 & 34.8 \\
9 & 3 & 3 & $\mathrm{I}$ & 66.1 \\
$\mathrm{~K} \mathrm{I}^{\mathrm{d}}(\%)$ & 48.6 & 25.6 & 45.7 & \\
$\mathrm{~K} 2(\%)$ & 47.9 & 39.2 & 48.3 & \\
$\mathrm{~K}^{2}(\%)$ & 43.3 & 75.0 & & \\
$\mathrm{R}^{\mathrm{e}}(\%)$ & 5.3 & 51.4 & 2.6 & \\
\hline
\end{tabular}

Notes: ${ }^{\mathrm{a}} \mathrm{A}$ is the hyaluronic acid solution concentration; ${ }^{\mathrm{B}} \mathrm{B}$ is the methylated collagen concentration; ${ }^{\mathrm{C} C}$ is the copolymer concentration; ${ }^{\mathrm{d}} \mathrm{K}$ is the average value of the factor; ${ }^{e} \mathrm{R}$ is the range. the optimal formulation. The optimized level for each factor was analyzed: A: $1>2>3$; B: $1>2>3$; C: $1>2$. The best combination for the formulation was chosen by combining the optimal levels of each factor. The optimal combination was A1B1C1, which is hyaluronic acid $(1 \mathrm{mg} / \mathrm{mL})$, methylated collagen $(1.5 \mathrm{mg} / \mathrm{mL})$, and terpolymer $(30 \mathrm{mg} / \mathrm{mL})$.

A burst release effect is that the cumulative drug release rate at the first 2 hours exceeds $30 \%-40 \%$ of the total amount of drug, so B3 was excluded for the formulation of prescription.

\section{Effects of each factor on the DOX release from the porous tantalum implants at 30 days}

According to the $\mathrm{R}$ values, three factors in the present experiment were ranged as $\mathrm{B}>\mathrm{A}>\mathrm{C}$ (Table 3). For more drug release from the functionalized implants, a higher Q2 is better for the optimal formulation. The optimized level for each factor was analyzed: A: $1>2>3$; B: $2>1 ; \mathrm{C}: 2>1$. The best combination for the formulation was chosen by combining the optimal levels of each factor. The optimal combination was A1B2C2, which is hyaluronic acid $(1 \mathrm{mg} / \mathrm{mL})$, methylated collagen $(3 \mathrm{mg} / \mathrm{mL})$, and terpolymer $(100 \mathrm{mg} / \mathrm{mL})$.

Taking the results from Tables 2 and 3 together, three factors in the present experiment were ranged as $\mathrm{B}>\mathrm{A}>\mathrm{C}$, which means that Factor $\mathrm{B}$ has the greatest impact on the cumulative drug release and Factor $\mathrm{C}$ has the least impact. For Factor A, it is the same level A1 as the optimal level for both indexes Q1 and Q2. For Factor B, there is no big difference between B1 and B2 for Q1. However, Q2 is too low at the B1 level. So the optimal formulation of prescription for the composite membrane is $\mathrm{A} 1 \mathrm{~B} 2 \mathrm{C} 2$, which is hyaluronic acid $(1 \mathrm{mg} / \mathrm{mL})$, methylated collagen $(3 \mathrm{mg} / \mathrm{mL})$, and terpolymer $(100 \mathrm{mg} / \mathrm{mL})$.

Through prescription optimization we can see that the DOX release rates were greatly affected by the concentrations of the three polyions. The concentration of methylated collagen has the highest effect on the drug release rate. Both DOX and methylated collagen are polycations. In this study we fixed the DOX loading amount at $100 \mu \mathrm{g}$, so concentration of methylated collagen has a significant effect and reacts with the other two negative charges: hyaluronic acid and terpolymer of HEMA-MMA-MAA.

Polyelectrolyte multilayer nanofilms have been widely used for surface modification of biomedical devices and drug delivery systems. The properties of the polyelectrolyte multilayer are controllable by selecting either different polyelectrolytes or different processing procedures. The choice of 
the assembly components is very flexible because many biological molecules are polyelectrolytes, (eg, nucleic acid, protein, glycoprotein, proteoglycan, glycosaminoglycans, alginates, and chitosan). The polyelectrolyte multilayer nanofilms are usually prepared by a layer-by-layer self-assembly technique to coat a device or substrate. By controlling the number of film layers, the concentration of the polyelectrolytes, and the drug incubation time, the drug loading and release from the polyelectrolyte multilayers are tunable.

With this versatile technique it is possible to build a multidrug release system to have a multifunction device. Different therapeutic agents can be loaded into different film layers. Taking an orthopedic implant as an example, prevention of implant infection and promoting tissue regeneration are the main tasks for implant fixation. Polyelectrolyte multilayer nanofilms with antibiotics, cytokines, or growth factors have been used to modify the biomedical devices for different aims.
The study has shown that interleukin 12 multilayer polypeptide nanoscale coatings at the implant/tissue interface substantially decreased infections in vivo. ${ }^{25}$ Recombinant human bone morphogenetic protein 2 (rhBMP-2) was delivered by crosslinked poly(L-lysine)/hyaluronan layer-by-layer films to induce myoblast differentiation to osteoblasts. ${ }^{26}$ Multifunctional implants could be manufactured by incorporating multiple drugs on the surface of implants via the processing of polyelectrolyte multilayer nanofilms. Such devices can use rhBMP-2 as the initial layer to induce osteogenic differentiation of mesenchymal stem cells, and use antibiotics such as gentamicin for the upper layer to prevent infection.

Based on our present study, we can also load rhBMP-2 as an initial layer, and anticancer drug DOX as the outside layer. This multifunctional implant could firstly release anticancer drug to prevent cancer recurrence and then promote bone tissue engineering.
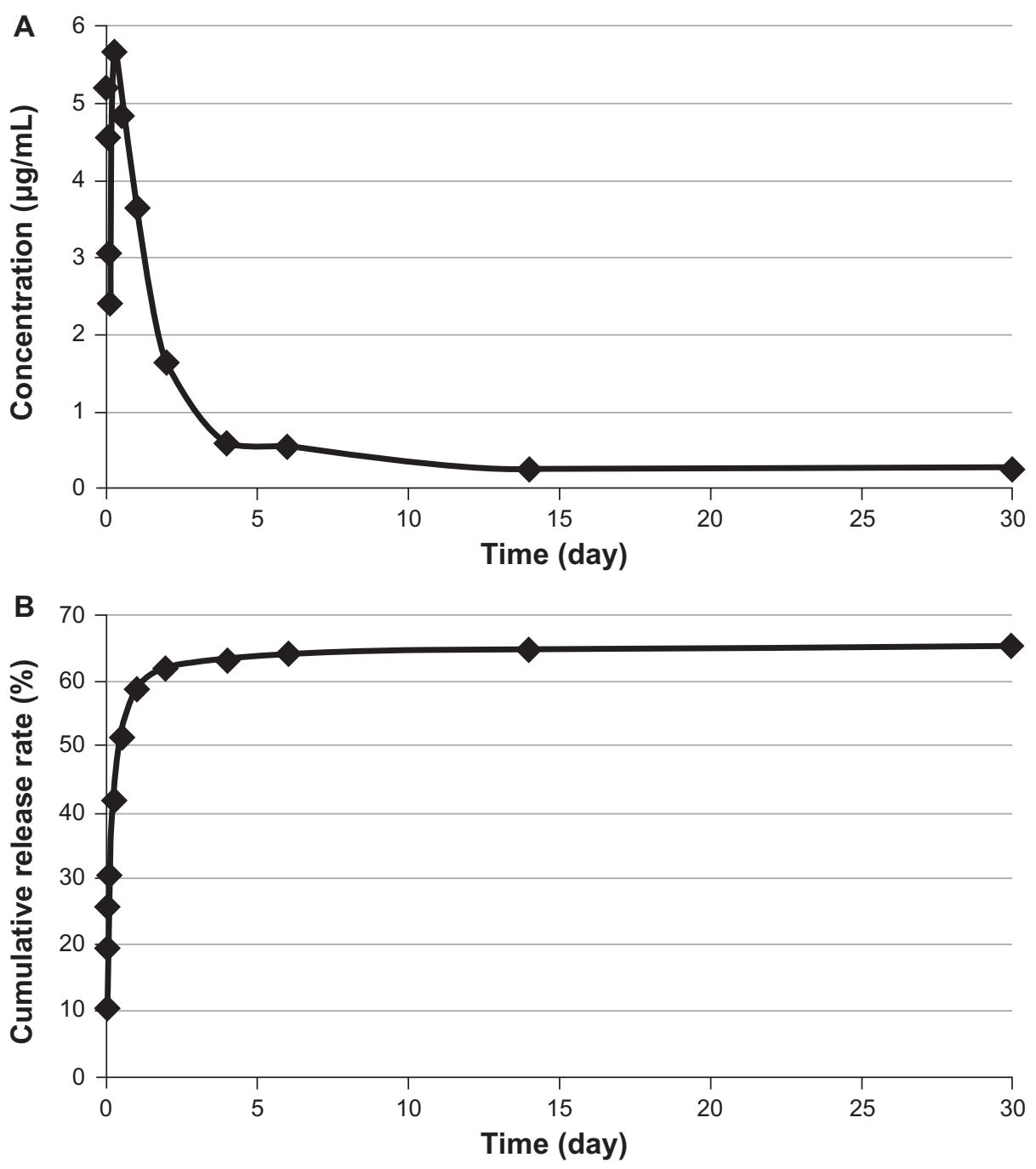

Figure 3 (A) Doxorubicin concentration released from functionalized porous tantalum implants at series time points. (B) Cumulative release rate of doxorubicin from functionalized porous tantalum implants in 30 days. 


\section{In vitro study of DOX release from functionalized porous tantalum implants}

DOX was loaded into the porous tantalum implants with the optimization of prescription. The DOX concentrations released from the functionalized porous tantalum implants at series time points and the cumulative release curve are depicted in Figure 3.

DOX released rapidly from the implants at the first 24 hours and then slowly sustained release for 30 days. The cumulative release curve was closed to zero-order rate from Day 2. We loaded $100 \mu \mathrm{g}$ of DOX in each functionalized porous tantalum implant, and the drug release concentrations ranged from $0.25 \mu \mathrm{g} / \mathrm{mL}$ to $5.64 \mu \mathrm{g} / \mathrm{mL}$ for 30 days. The highest concentration of the release solutions was $5.64 \mu \mathrm{g} / \mathrm{mL}$ at 6 hours. A release concentration greater than $0.25 \mu \mathrm{g} / \mathrm{mL}$ was maintained.

Drug release rates from polyelectrolyte multilayers depend on the polyelectrolytes applied in the drug delivery system. Drug release mechanisms are different. They can be induced by charge, temperature, degradation of polyelectrolyte multilayer, hydrolysis, or redox..$^{27}$ Many studies have been trying to control drug release rates by controlling the release environment $\mathrm{pH},{ }^{28}$ the temperature, ${ }^{29}$ or the construction of the polyelectrolyte multilayer. ${ }^{30}$

In the present study, there are two phases of drug release rates: an initial burst release and a sustained release. The initial burst release on the first day is mainly caused by the absorption of the free DOX, which is not encapsulated into the copolymeric membranes but physically absorbed on the implant. The second phase of sustained release is mainly due to the degradation of hyaluronic acid, which induces the change of net charges of polyelectrolytes and releases the DOX. DOX release from the implant may also involve a diffusion mechanism, where DOX diffuses from the higher concentration of the implant to the release buffer.

In clinical practice, DOX is given with a dose of $60 \mathrm{mg} / \mathrm{m}^{2}$ every 3 weeks. It is reported that DOX reaches peak plasma concentration at $2.7 \mu \mathrm{g} / \mathrm{mL} .{ }^{31}$ The implantable drug delivery system in the present study shows the high efficiency of the loaded drug, with a satisfactory sustained-release capability.

A study by Itokazu et a ${ }^{32}$ showed that a local drug delivery system has the advantage of minimizing systemic side effects and has long-term localized delivery of anticancer drug. They showed that the release of DOX was sustained for 66 days in vitro and 4 weeks in vivo. DOX concentrations in plasma, liver, and kidney ranged from $0.25 \%$ to $10 \%$ of that at the implanted site. However, in their system, the hydroxyapatite blocks did not have adequate mechanical support for the bone-bearing part.

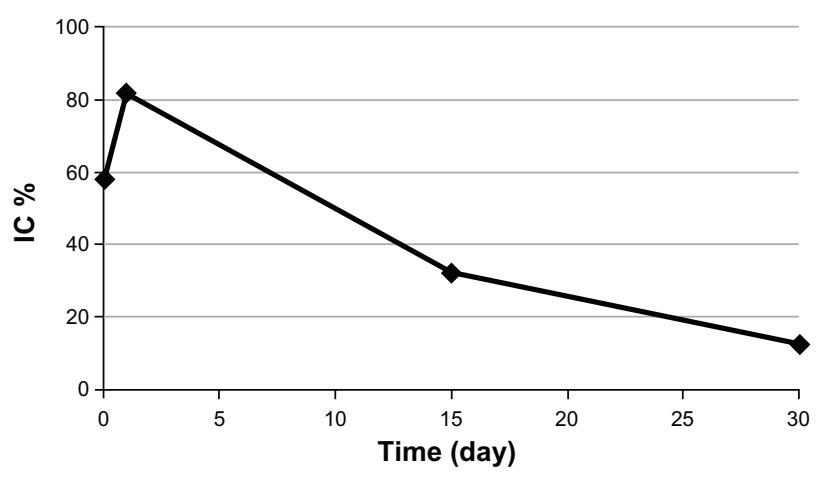

Figure 4 Inhibitory effects of doxorubicin release solutions on the growth of chondrosarcoma cell line SWI353. Data represent the mean \pm SD $(n=6)$.

\section{Cytotoxicity of DOX release solutions from the functionalized porous tantalum implants}

Figure 4 shows that DOX release solutions from the porous tantalum implants in 30 days all had an inhibition effect on the growth of chondrosarcoma cells. The inhibitory ratios of DOX release solutions from 2 hours, 24 hours, 15 days, and 30 days were $57.87 \%, 81.42 \%, 31.78 \%$, and $12.19 \%$, respectively.

These results proved that the drug loading process did not change the bioactivity of DOX. When the implant is used as a drug delivery vehicle, it is very important to consider the interaction between the material and the loaded drug. The implant should not have any effects on the loaded drug and also be able to protect the stability and bioactivity of the loaded drug.

\section{Conclusion}

This study investigated a modification procedure to incorporate an anticancer drug into a widely clinically used porous tantalum implant in order to have an implantable drug delivery system. Polyelectrolytes of hyaluronic acid, methylated collagen, and terpolymer of HEMA-MMA-MAA were electrostatically self-assembled on the surface of the porous tantalum implants. We optimized three factors of the self-assembly membranes by orthogonal experimental design and obtained the optimal drug release of the loaded drug DOX. Our results showed that the functionalized porous tantalum implants loaded with DOX had a stable and sustained drug release for 30 days and that the drug release solutions all had inhibitory effects on the growth of chondrosarcoma cell SW1353.

Base on the aforementioned results, it is predicted that this drug-loaded porous tantalum implant could be placed into the surgical defect created by bone tumor resection for immediate load-bearing support and local anticancer treatment to 
prevent recurrence. The functionalized technique in the present study can also apply to different types of implants to have different local drug delivery systems for various therapeutic aims. Thus, further in vivo experiments should be proposed in the future.

\section{Acknowledgments}

This work was supported by the National Natural Science Foundation of China (No. 30571892, u0732001) and the Danish Research Council (Jr.nr. 09-063120). Type I bovine collagen was supplied courtesy of Professor Yingjun Wang, the Key Lab for Special Functional Materials of the Ministry of Education, South China University of Technology, China.

\section{Disclosure}

The authors disclose no conflicts of interest in this work.

\section{References}

1. Dubousset J. Strategy for the surgical treatment of primary bone tumors of the spine in children. Chir Organi Mov. 1990;75(1 Suppl):89-90.

2. Dragan S, Wall A, Krawczyk A, et al. Local experience in the surgical treatment of primary malignant and metastatic bone tumors of the proximal femur. Ortop Traumatol Rehabil. 2005;7(6):604-610.

3. Niinomi M. Metallic biomaterials. J Artif Organs. 2008;11(3):105-110.

4. Levine BR, Sporer S, Poggie RA, et al. Experimental and clinical performance of porous tantalum in orthopedic surgery. Biomaterials. 2006;27(27):4671-4681.

5. Bobyn JD, Toh KK, Hacking SA, et al. Tissue response to porous tantalum acetabular cups: a canine model. J Arthroplasty. 1999;14(3): 347-354.

6. Zou X, Li H, Bünger M, et al. Bone ingrowth characteristics of porous tantalum and carbon fiber interbody devices: an experimental study in pigs. Spine J. 2004;4(1):99-105.

7. Long M, Rack HJ. Titanium alloys in total joint replacement: a materials science perspective. Biomaterials. 1998;19(18):1621-1639.

8. Bobyn JD, Stackpool GJ, Hacking SA, et al. Characteristics of bone in growth and interface mechanics of a new porous tantalum biomaterial. J Bone Joint Surg Br. 1999;81(5):907-914.

9. Alberius P. Bone reactions to tantalum markers. A scanning electron microscopic study. Acta Anat (Basel). 1983;115(4):310-318.

10. Aronson AS, Hansson LI. Effect of tantalum markers of longitudinal bone growth. Acta Orthop Scand. 1976;47(5):515-519.

11. Patil N, Lee K, Goodman SB. Porous tantalum in hip and knee reconstructive surgery. J Biomed Mater Res Part B Appl Biomater. 2009;89(1):242-251.

12. Fröschle GW, Mählitz J, Langendorff HU, et al. Release of daunorubicin from polymethylmethacrylate for the improvement of the local growth control of bone metastasis animal experiments. Anticancer Res. 1997;17(2A):995-1002.

13. El-Ghannam A, Ricci K, Malkawi A, et al. A ceramic-based anticancer drug delivery system to treat breast cancer. J Mater Sci Mater Med. 2010;21(9):2701-2710.

International Journal of Nanomedicine

\section{Publish your work in this journal}

The International Journal of Nanomedicine is an international, peerreviewed journal focusing on the application of nanotechnology in diagnostics, therapeutics, and drug delivery systems throughout the biomedical field. This journal is indexed on PubMed Central, MedLine, CAS, SciSearch ${ }^{\circledR}$, Current Contents ${ }^{\circledR} /$ Clinical Medicine,
14. Porter J, Ruckh T, Popat KC. Bone tissue engineering: a review in bone biomimetics and drug delivery strategies. Biotechnol Progress. 2009;25(6):1539-1560

15. Defail AJ, Edington HD, Matthews S, et al. Controlled release of bioactive doxorubicin from microspheres embedded within gelatin scaffolds. J Biomed Mater Res. 2006;79(4):954-962.

16. Chen M, Le D, Baatrup A, et al. Self-assembled composite matrix in a hierarchical 3-D scaffold for bone tissue engineering. Acta Biomaterialia. 2011:1-13.

17. Chia SM, Leong KW, Li J, et al. Hepatocyte encapsulation for enhanced cellular functions. Tissue Eng. 2000;6(5):481-495.

18. Zhang J, Wei HP, Quek CH, et al. Quantitative measurement of collagen methylation by capillary electrophoresis. Electrophoresis. 2004;25(20):3416-3421.

19. Lankalapalli S, Kolapalli VR. Polyelectrolyte complexes: a review of their applicability in drug delivery technology. Indian J Pharm Sci. 2009;71(5):481-487.

20. Sun T, Chan ML, Zhou Y, et al. Use of ultrathin shell microcapsules of hepatocytes in bioartificial liver-assist device. Tissue Eng. 2003;9 Suppl 1: S65-S75.

21. Quek CH, Li J, Sun T, et al. Photo-crosslinkable microcapsules formed by polyelectrolyte copolymer and modified collagen for rat hepatocyte encapsulation. Biomaterials. 2004;25(17):3531-3540.

22. Miyazaki T, Kim HM, Miyaji F, et al. Bioactive tantalum metal prepared by $\mathrm{NaOH}$ treatment. J Biomed Mater Res. 2000;50(1):35-42.

23. Toh YC, Ho ST, Zhou Y, et al. Application of a polyelectrolyte complex coacervation method to improve seeding efficiency of bone marrow stromal cells in a 3D culture system. Biomaterials. 2005;26(19): 4149-4160.

24. Liao IC, Wan AC, Yim EK, Leong KW. Controlled release from fibers of polyelectrolyte complexes. J Control Release. 2005;104(2): 347-358.

25. Li B, Jiang B, Boyce BM, Lindsey BA. Multilayer polypeptide nanoscale coatings incorporating IL-12 for the prevention of biomedical device-associated infections. Biomaterials. 2009;30(13): 2552-2558.

26. Crouzier T, Ren K, Nicolas C, et al. Layer-by-layer films as a biomimetic reservoir for rhBMP-2 delivery: controlled differentiation of myoblasts to osteoblasts. Small. 2009;5(5):598-608

27. Jiang B, Barnett JB, Li B. Advances in polyelectrolyte multilayer nanofilms as tunable drug delivery systems. Nanotechnol Sci Appl. 2009;2:21-27.

28. Jiang B, Li B. Tunable drug loading and release from polypeptide multilayer nanofilms. Int J Nanomedicine. 2009;4:37-53.

29. Quinn JF, Caruso F. Thermoresponsive nanoassemblies: layer-bylayer assembly of hydrophilic-hydrophobic alternating copolymers. Macromolecules. 2005;38:3414-3419.

30. Berg MC, Zhai L, Cohen RE, Rubner MF. Controlled drug release from porous polyelectrolyte multilayers. Biomacromolecules 2006;7(1): 357-364.

31. Müller I, Jenner A, Bruchelt G, et al. Effect of concentration on the cytotoxic mechanism of doxorubicin - apoptosis and oxidative DNA damage. Biochem Biophys Res Commun. 1997;230(2):254-257.

32. Itokazu M, Kumazawa S, Wada E, Wenyi Y. Sustained release of adriamycin from implanted hydroxyapatite blocks for the treatment of experimental osteogenic sarcoma in mice. Cancer Lett. 1996;107(1): $11-18$.

\section{Dovepress}

Journal Citation Reports/Science Edition, EMBase, Scopus and the Elsevier Bibliographic databases. The manuscript management system is completely online and includes a very quick and fair peer-review system, which is all easy to use. Visit http://www.dovepress.com/ testimonials.php to read real quotes from published authors. 